\title{
Almost Periodic Solution of a Modified Leslie-Gower Predator-Prey Model with Beddington-DeAngelis Functional Response and Feedback Controls
}

\author{
Kerong Zhang, ${ }^{1}$ Jianli Li, ${ }^{1}$ and Aiwen $\mathrm{Yu}^{2}$ \\ ${ }^{1}$ Department of Mathematics, Hunan Normal University, Changsha, Hunan 410081, China \\ ${ }^{2}$ Department of Mathematics, Beiya Middle School, Changsha, Hunan 410008, China \\ Correspondence should be addressed to Jianli Li; ljianli@sina.com
}

Received 30 January 2014; Revised 19 February 2014; Accepted 26 February 2014; Published 22 April 2014

Academic Editor: Bingwen Liu

Copyright (C) 2014 Kerong Zhang et al. This is an open access article distributed under the Creative Commons Attribution License, which permits unrestricted use, distribution, and reproduction in any medium, provided the original work is properly cited.

We consider a modified Leslie-Gower predator-prey model with the Beddington-DeAngelis functional response and feedback controls as follows: $\dot{x}(t)=x(t)\left(a_{1}(t)-b(t) x(t)-c(t) y(t) /(\alpha(t)+\beta(t) x(t)+\gamma(t) y(t))-e_{1}(t) u(t)\right), \dot{u}(t)=-d_{1}(t) u(t)+$ $p_{1}(t) x(t-\tau), \dot{y}(t)=y(t)\left(a_{2}(t)-r(t) y(t) /(x(t)+k(t))-e_{2}(t) \nu(t)\right)$, and $\dot{\gamma}(t)=-d_{2}(t) v(t)+p_{2}(t) y(t-\tau)$. Sufficient conditions which guarantee the permanence and existence of a unique globally attractive positive almost periodic solution of the system are obtained.

\section{Introduction}

In recent years, the modified predator-prey systems with periodic or almost periodic coefficients have been studied extensively.

Leslie [1] proposed the famous Leslie predator-prey system as follows:

$$
\begin{gathered}
\dot{x}(t)=x(a-b x)-p(x) y, \\
\dot{y}=y\left(e-f \frac{y}{x}\right),
\end{gathered}
$$

where $x$ and $y$ stand for the population of the prey and the predator at time $t$, respectively, and $p(x)$ is the so-called predator functional response to the prey. The term $y / x$ is the Leslie-Gower term which measures the loss in the predator population due to rarity of its favorite food.

Global stability of the positive locally asymptotically stable equilibrium in a class of predator-prey systems has been introduced by Hsu and Huang [2], and the system is as follows:

$$
\frac{d x}{d t}=r x\left(1-\frac{x}{k}-y p(x)\right)
$$

$$
\begin{gathered}
\frac{d y}{d x}=y\left[s\left(1-\frac{h y}{s}\right)\right], \\
x(0)>0, \quad y(0)>0, \quad r, s, k, h>0 .
\end{gathered}
$$

When the functional response $p(x)$ equals $m x$, then (2) turns into a Leslie-Gower system [3].

On the other hand, the periodic solution (almost periodic solution) and some other properties of Leslie-Gower predator-prey models were studied (see [4-9]). In particular, Zhang [10] discussed the almost periodic solution of a modified Leslie-Gower predator-prey model with the BeddingtonDeAngelis function response as follows:

$$
\begin{gathered}
\dot{x}(t)=x(t)\left(r_{1}(t)-b(t) x(t)\right. \\
\left.-\frac{c(t) y(t)}{\alpha(t)+\beta(t) x(t)+\gamma(t) y(t)}\right), \\
\dot{y}(t)=y(t)\left(r_{2}(t)-\frac{d(t) y(t)}{x(t)+k(t)}\right),
\end{gathered}
$$

where $x(t)$ is the size of prey population and $y(t)$ is the size of predator population. 
Stimulated by the above reasons, in this paper, we incorporate the feedback control into model (3) and consider the following model:

$$
\begin{gathered}
\dot{x}(t)=x(t)\left(a_{1}(t)-b(t) x(t)\right. \\
\left.\quad-\frac{c(t) y(t)}{\alpha(t)+\beta(t) x(t)+\gamma(t) y(t)}-e_{1}(t) u(t)\right), \\
\dot{u}(t)=-d_{1}(t) u(t)+p_{1}(t) x(t-\tau), \\
\dot{y}(t)=y(t)\left(a_{2}(t)-\frac{r(t) y(t)}{x(t)+k(t)}-e_{2}(t) v(t)\right), \\
\dot{v}(t)=-d_{2}(t) v(t)+p_{2}(t) y(t-\tau),
\end{gathered}
$$

where $\tau>0$ and all the coefficients $b(t), c(t), r(t), k(t)$, $\alpha(t), \beta(t), \gamma(t), a_{i}(t), d_{i}(t), p_{i}(t)$, and $e_{i}(t)(i=1,2)$ are all continuous, almost periodic functions on $R$.

Associated with (4), we consider a group of initial conditions with the following form (we assume, without loss of generality, that the initial time $\left.t_{0}=0\right)$ :

$$
\begin{gathered}
x(s)=\phi(s) \geq 0, \quad s \in[-\tau, 0], \phi(0)>0, \\
y(s)=\varphi(s) \geq 0, \quad s \in[-\tau, 0], \varphi(0)>0, \\
u(0)>0, \quad v(0)>0 .
\end{gathered}
$$

Let $f$ be a continuous bounded function on $R$ and we set

$$
f^{l}=\inf _{t \in R} f(t), \quad f^{u}=\sup _{t \in R} f(t) .
$$

Throughout this paper, we assume that the coefficients of the almost periodic system (4) satisfy

$$
\begin{gathered}
\min _{i=1,2}\left\{b^{l}, c^{l}, \alpha^{l}, \beta^{l}, \gamma^{l}, r^{l}, k^{l}, a_{i}^{l}, d_{i}^{l}, p_{i}^{l}, e_{i}^{l}\right\}>0, \\
\max _{i=1,2}\left\{b^{u}, c^{u}, \alpha^{u}, \beta^{u}, \gamma^{u}, r^{u}, k^{u}, a_{i}^{u}, d_{i}^{u}, p_{i}^{u}, e_{i}^{u}\right\}<+\infty .
\end{gathered}
$$

By constructing a suitable Lyapunov functional, we obtain some sufficient conditions for the existence of a globally attractive positive almost periodic solution of system (4) with initial conditions (5).

\section{Permanence}

In this section, we give some definitions and results that we will use in the rest of the paper.

Lemma 1 (see [11]). If $a>0, b>0$, and $\dot{x} \geq(\leq) x(b-a x)$, when $t \geq 0$ and $x(0)>0$, one has

$$
\liminf _{t \rightarrow+\infty} x(t) \geq \frac{b}{a}, \quad\left(\limsup _{t \rightarrow+\infty} x(t) \leq \frac{b}{a}\right) .
$$

Lemma 2 (see [11]). If $a>0, b>0$, and $\dot{x} \geq(\leq) b-a x$, when $t \geq 0$ and $x(0)>0$, one has

$$
\liminf _{t \rightarrow+\infty} x(t) \geq \frac{b}{a}, \quad\left(\limsup _{t \rightarrow+\infty} x(t) \leq \frac{b}{a}\right) .
$$

Set the following:

$$
\begin{gathered}
M_{1}=\frac{a_{1}^{u}}{b^{l}}, \quad L_{1}=\frac{p_{1}^{u} M_{1}}{d_{1}^{l}}, \\
M_{2}=\frac{a_{2}^{u}\left(M_{1}+k^{u}\right)}{r^{l}}, \quad L_{2}=\frac{p_{2}^{u} M_{2}}{d_{2}^{l}}, \\
m_{1}=\frac{a_{1}^{l}-c^{u} / r^{l}-e_{1}^{u} L_{1}}{b^{u}}, \quad l_{1}=\frac{p_{1}^{l} m_{1}}{d_{1}^{u}}, \\
m_{2}=\frac{1}{r^{u}}\left(a_{2}^{l}-e_{2}^{u} L_{2}\right)\left(m_{1}+k^{l}\right), \quad l_{2}=\frac{p_{2}^{l} m_{2}}{d_{2}^{u}} .
\end{gathered}
$$

Theorem 3. Suppose that system (4) with initial condition (5) satisfies the following condition:

$$
a_{1}^{l}-\frac{c^{u}}{r^{l}}-e_{1}^{u} L_{1}>0, \quad a_{2}^{l}-e_{2}^{u} L_{2}>0 .
$$

Then system (4) is permanent; that is, any positive solution $(x(t), u(t), y(t), v(t))^{T}$ of the system (4) satisfies

$$
\begin{gathered}
0<m_{1} \leq \liminf _{t \rightarrow+\infty} x(t) \leq \limsup _{t \rightarrow+\infty} x(t) \leq M_{1}, \\
0<l_{1} \leq \liminf _{t \rightarrow+\infty} u(t) \leq \limsup _{t \rightarrow+\infty} u(t) \leq L_{1}, \\
0<m_{2} \leq \liminf _{t \rightarrow+\infty} y(t) \leq \limsup _{t \rightarrow+\infty} y(t) \leq M_{2}, \\
0<l_{2} \leq \liminf _{t \rightarrow+\infty} v(t) \leq \limsup _{t \rightarrow+\infty} v(t) \leq L_{2} .
\end{gathered}
$$

Proof. From the first equation of (4), we have the following:

$$
\dot{x}(t) \leq x(t)\left(a_{1}^{u}-b^{l} x(t)\right) .
$$

Applying Lemma 1 to (13) leads to

$$
\limsup _{t \rightarrow+\infty} x(t) \leq \frac{a_{1}^{u}}{b^{l}}=M_{1}
$$

From (14), we know that there exists an enough large $T_{1}>0$ such that

$$
x(t) \leq M_{1}, \quad t \geq T_{1}>0
$$

so there exists an enough large $T_{2}=T_{1}+\tau$ such that

$$
x(t-\tau) \leq M_{1}, \quad t \geq T_{2}>0 .
$$

It follows from (16) and the second equation of system (4) that, for $t \geq T_{2}$,

$$
\dot{u}(t) \leq-d^{l} u(t)+p_{1}^{u} M_{1} .
$$

Applying Lemma 2 to (17) leads to

$$
\limsup _{t \rightarrow+\infty} u(t) \leq \frac{p_{1}^{u} M_{1}}{d_{1}^{l}}=L_{1}
$$


By using a similar argument as that in the proof of (14) and (18), we can get the following:

$$
\begin{gathered}
\limsup _{t \rightarrow+\infty} y(t) \leq \frac{a_{2}^{u}\left(M_{1}+k^{u}\right)}{r^{l}}=M_{2}, \\
\limsup _{t \rightarrow+\infty} v(t) \leq \frac{p_{2}^{u} M_{2}}{d_{2}^{l}}=L_{2} .
\end{gathered}
$$

From (18) and the first equation of system (4) we know

$$
\dot{x}(t) \geq x(t)\left(a_{1}^{l}-\frac{c^{u}}{\gamma^{l}}-e_{1}^{u} L_{1}-b^{u} x(t)\right) .
$$

Applying Lemma 1 and (11) to the above leads to

$$
\liminf _{t \rightarrow+\infty} x(t) \geq \frac{a_{1}^{l}-c^{u} / r^{l}-e_{1}^{u} L_{1}}{b^{u}}=m_{1} .
$$

Therefore, we know that there exists an enough large $T_{3}$ such that

$$
x(t) \geq m_{1}, \quad t \geq T_{3}>0 .
$$

From the second equation of system (4) we have the following:

$$
\dot{u}(t) \geq-d_{1}^{u} u(t)+p_{1}^{l} m_{1} .
$$

Applying Lemma 2 to the above, we obtain the following:

$$
\liminf _{t \rightarrow+\infty} u(t) \geq \frac{p_{1}^{l} m_{1}}{d_{1}^{u}}=l_{1} .
$$

By using a similar method as that in the proof of (21) and (24), it follows that

$$
\begin{aligned}
& \liminf _{t \rightarrow+\infty} y(t) \geq \frac{1}{r^{u}}\left(a_{2}^{l}-e_{2}^{u} L_{2}\right)\left(m_{1}+k^{l}\right)=m_{2} \\
& \liminf _{t \rightarrow+\infty} \nu(t) \geq \frac{p_{2}^{l} m_{2}}{d_{2}^{u}}=l_{2} .
\end{aligned}
$$

This completes the proof.

We denote by $\Omega$ the set of all solutions $z(t)=(x(t), u(t)$, $y(t), v(t))^{T}$ of system (4) satisfying $m_{1} \leq x(t) \leq M_{1}, l_{1} \leq$ $u(t) \leq L_{1}, m_{2} \leq y(t) \leq M_{2}$, and $l_{2} \leq v(t) \leq L_{2}$ for all $t>0$.

Theorem 4. Consider the following: $\Omega \neq \emptyset$.

Proof. From the properties of almost periodic function there exists a sequence $\left\{t_{n}\right\}$ with $t_{n} \rightarrow+\infty$ as $n \rightarrow+\infty$ such that

$$
\begin{aligned}
& a_{i}\left(t+t_{n}\right) \longrightarrow a_{i}(t), \quad d_{i}\left(t+t_{n}\right) \longrightarrow d_{i}(t), \\
& e_{i}\left(t+t_{n}\right) \longrightarrow e_{i}(t), \quad p_{i}\left(t+t_{n}\right) \longrightarrow p_{i}(t), \\
& (i=1,2), \\
& b\left(t+t_{n}\right) \longrightarrow b(t), \quad c\left(t+t_{n}\right) \longrightarrow c(t), \\
& r\left(t+t_{n}\right) \longrightarrow r(t), \quad k\left(t+t_{n}\right) \longrightarrow k(t), \\
& \alpha\left(t+t_{n}\right) \longrightarrow \alpha(t), \quad \beta\left(t+t_{n}\right) \longrightarrow \beta(t), \\
& \gamma\left(t+t_{n}\right) \longrightarrow \gamma(t),
\end{aligned}
$$

as $n \rightarrow \infty$ uniformly on $R$. Let $z(t)=(x(t), u(t), y(t), v(t))^{T}$ be a solution of system (4) satisfying $m_{1} \leq x(t) \leq M_{1}$, $l_{1} \leq u(t) \leq L_{1}, m_{2} \leq y(t) \leq M_{2}$, and $l_{2} \leq v(t) \leq L_{2}$ for $t>T$. Clearly, the sequence $z\left(t+t_{n}\right)$ is uniformly bounded and equicontinuous on each bounded subset of $R$. Therefore, by the Arzelà-Ascoli theorem, there exists a subsequence $z\left(t+t_{k}\right)$ which converges to a continuous function $z^{*}(t)=$ $\left(x^{*}(t), u^{*}(t), y^{*}(t), v^{*}(t)\right)^{T}$ as $k \rightarrow+\infty$ uniformly on each bounded subset of $R$. Let $T_{0} \in R$ be given. We may assume that $t_{k}+T_{0} \geq T$ for all $k$. For $t \geq 0$, we have the following:

$$
\begin{aligned}
& x\left(t+t_{k}+T_{0}\right) \\
& =x\left(t_{k}+T_{0}\right) \\
& +\int_{T_{0}}^{t+T_{0}} x\left(s+t_{k}\right)\left(a_{1}\left(s+t_{k}\right)-b\left(s+t_{k}\right) x\left(s+t_{k}\right)\right. \\
& -\left(c\left(s+t_{k}\right) y\left(s+t_{k}\right)\right) \\
& \times\left(\alpha\left(s+t_{k}\right)+\beta\left(s+t_{k}\right) x\left(s+t_{k}\right)\right. \\
& \left.\quad+\gamma\left(s+t_{k}\right) y\left(s+t_{k}\right)\right)^{-1} \\
& \left.-e_{1}\left(s+t_{k}\right) u\left(s+t_{k}\right)\right) d s,
\end{aligned}
$$

$$
\begin{aligned}
& u\left(t+t_{k}+T_{0}\right) \\
& =u\left(t_{k}+T_{0}\right) \\
& -\int_{T_{0}}^{t+T_{0}} d_{1}\left(s+t_{k}\right) u\left(s+t_{k}\right)+p_{1}\left(s+t_{k}\right) x\left(s+t_{k}-\tau\right) d s, \\
& y\left(t+t_{k}+T_{0}\right) \\
& =y\left(t_{k}+T_{0}\right) \\
& +\int_{T_{0}}^{t+T_{0}} y\left(s+t_{k}\right)\left(a_{2}\left(s+t_{k}\right)-\frac{r\left(s+t_{k}\right) y\left(s+t_{k}\right)}{x\left(s+t_{k}\right)+k\left(s+t_{k}\right)}\right. \\
& \left.-e_{2}\left(s+t_{k}\right) v\left(s+t_{k}\right)\right) d s \\
& \dot{v}\left(t+t_{k}+T_{0}\right) \\
& =v\left(t_{k}+T_{0}\right) \\
& +\int_{T_{0}}^{t+T_{0}}-d_{2}\left(s+t_{k}\right) v\left(s+t_{k}\right)+p_{2}\left(s+t_{k}\right) y\left(s+t_{k}-\tau\right) d s .
\end{aligned}
$$

Applying Lebesgue's dominated convergence theorem and letting $k \rightarrow+\infty$ in (27), we obtain the following:

$$
\begin{aligned}
& x^{*}\left(t+T_{0}\right) \\
& =x^{*}\left(T_{0}\right) \\
& +\int_{T_{0}}^{t+T_{0}} x^{*}(s)\left(a_{1}(s)-b(s) x^{*}(s)\right. \\
& -\frac{c(s) y^{*}(s)}{\alpha(s)+\beta(s) x^{*}(s)+\gamma(s) y^{*}(s)} \\
& \left.-e_{1}(s) u^{*}(s)\right) d s,
\end{aligned}
$$




$$
\begin{aligned}
u^{*}\left(t+T_{0}\right)= & u^{*}\left(T_{0}\right) \\
& -\int_{T_{0}}^{t+T_{0}} d_{1}(s) u^{*}(s)+p_{1}(s) x^{*}(s-\tau) d s, \\
y^{*}\left(t+T_{0}\right)= & y^{*}\left(T_{0}\right) \\
& +\int_{T_{0}}^{t+T_{0}} y^{*}(s)\left(a_{2}(s)-\frac{r(s) y^{*}(s)}{x^{*}(s)+k(s)}\right. \\
v^{*}\left(t+T_{0}\right)= & v^{*}\left(T_{0}\right) \\
& +\int_{T_{0}}^{t+T_{0}}-d_{2}(s) v^{*}(s)+p_{2}(s) y^{*}(s-\tau) d s .
\end{aligned}
$$

Since $T_{0} \in R$ is arbitrarily given, $z^{*}(t)=\left(x^{*}(t), u^{*}(t), y^{*}(t)\right.$, $\left.v^{*}(t)\right)^{T}$ is a solution of system (4) on $R$. It is clear that $m_{1} \leq$ $x^{*}(t) \leq M_{1}, l_{1} \leq u^{*}(t) \leq L_{1}, m_{2} \leq y^{*}(t) \leq M_{2}, l_{2} \leq v^{*}(t) \leq$ $L_{2}$ for $t \in R$. Thus $z^{*}(t) \in \Omega$. This completes the proof.

\section{Existence of a Unique Almost Periodic Solution}

Now let us state several definitions and lemmas which will be useful in the proving of the main result of this section.

Definition 5 (see [12]). A function $f(t, x)$, where $f$ is an $m$-vector, $t$ is a real scalar, and $x$ is an $n$-vector, is said to be almost periodic in $t$ uniformly with respect to $x \in S \subset R^{n}$, if $f(t, x)$ is continuous in $t \in R$ and $x \in S$ and if, for any $\varepsilon>0$, there is a constant $l(\varepsilon)>0$ such that in any interval of length $l(\varepsilon)$ there exists a $\varsigma$ such that the inequality

$$
|f(t+\varsigma, x)-f(t, x)|<\varepsilon
$$

is satisfied for all $t \in(-\infty,+\infty), x \in S$. The number $\varsigma$ is called an $\varepsilon$-translation number of $f(t, x)$.

Definition 6 (see [12]). A function $f: R \rightarrow R$ is said to be asymptotically almost periodic function, if there exists an almost periodic function $q(t)$ and a continuous function $r(t)$ such that $f(t)=q(t)+r(t), t \in R$ and $r(t) \rightarrow 0$ as $t \rightarrow \infty$.

Lemma 7 (see [13]). Let $f$ be a nonnegative, integral, and uniformly continuous function defined on $[0,+\infty)$; then $\lim _{t \rightarrow+\infty} f(t)=0$.

Theorem 8. Suppose that all conditions of Theorem 3 hold; furthermore assume that
$(H) \Theta>0$, where $\Theta=\min \left\{\Theta_{1}, \Theta_{2}, \Theta_{3}, \Theta_{4}\right\}$,

$$
\begin{aligned}
& \Theta_{1}= b^{l} m_{1}-p_{1}^{u} M_{1}-\frac{c^{u} \beta^{u} M_{1} M_{2}}{\left(\alpha^{l}+\beta^{l} m_{1}+\gamma^{l} m_{2}\right)^{2}} \\
&-\frac{r^{u} M_{1} M_{2}}{\left(m_{1}+k^{l}\right)^{2}}>0, \\
& \Theta_{2}= \frac{\gamma^{l}}{M_{1}+k^{u}}-\frac{c^{l} m_{2}}{\alpha^{u}+\beta^{u} M_{1}+\gamma^{u} M_{2}} \\
&-\frac{c^{u} \gamma^{u} M_{2}^{2}}{\left(\alpha^{l}+\beta^{l} m_{1}+\gamma^{l} m_{2}\right)^{2}}-p_{2}^{u} M_{2}>0, \\
& \Theta_{3}=d_{1}^{l}-e_{1}^{u}, \quad \Theta_{4}=d_{2}^{l}-e_{2}^{u} .
\end{aligned}
$$

Then system (4) with initial conditions (5) is globally attractive.

Proof. Let $x(t)=e^{x_{1}(t)}, y(t)=e^{y_{1}(t)}$, and then system (4) is transformed into

$$
\begin{aligned}
\dot{x}_{1}(t)= & a_{1}(t)-b(t) e^{x_{1}(t)} \\
& -\frac{c(t) e^{y_{1}(t)}}{\alpha(t)+\beta(t) e^{x_{1}(t)}+\gamma(t) e^{y_{1}(t)}}-e_{1}(t) u(t), \\
\dot{u}(t)= & -d_{1}(t) u(t)+p_{1}(t) e^{x_{1}(t-\tau)}, \\
\dot{y}_{1}(t)= & a_{2}(t)-\frac{r(t) e^{y_{1}(t)}}{e^{x_{1}(t)}+k(t)}-e_{2}(t) v(t), \\
\dot{v}(t)= & -d_{2}(t) v(t)+p_{2}(t) e^{y_{1}(t-\tau)} .
\end{aligned}
$$

Suppose that $z_{1}(t)=\left(x_{1}(t), u(t), y_{1}(t), v(t)\right)^{T}$ and $z_{1}^{*}(t)=$ $\left(x_{1}^{*}(t), u^{*}(t), y_{1}^{*}(t), v^{*}(t)\right)^{T}$ are any two positive solutions of (31).

$$
\begin{aligned}
& \text { Let } V(t)=V_{1}(t)+V_{2}(t)+V_{3}(t)+V_{4}(t) \text {, where } \\
& \qquad V_{1}(t)=\left|x_{1}(t)-x_{1}^{*}(t)\right|, \\
& V_{2}(t)=\left|u(t)-u^{*}(t)\right|+p_{1}^{u} \int_{t-\tau}^{t}\left|e^{x_{1}(t)}-e^{x_{1}^{*}(t)}\right| d s, \\
& V_{3}(t)=\left|y_{1}(t)-y_{1}^{*}(t)\right|, \\
& V_{4}(t)=\left|v(t)-v^{*}(t)\right|+p_{2}^{u} \int_{t-\tau}^{t}\left|e^{y_{1}(t)}-e^{y_{1}^{*}(t)}\right| d s .
\end{aligned}
$$

Calculating the right derivative $D^{+} V_{1}(t)$ of $V_{1}(t)$ along the solution of (31), we have the following:

$$
\begin{aligned}
D^{+} V_{1}(t)= & \operatorname{sgn}\left(x_{1}(t)-x_{1}^{*}(t)\right) \\
\times\left[-b(t)\left(e^{x_{1}(t)}-e^{x_{1}^{*}(t)}\right)\right. & -\frac{c(t) e^{y_{1}(t)}}{\alpha(t)+\beta(t) e^{x_{1}(t)}+\gamma(t) e^{y_{1}(t)}} \\
& +\frac{c(t) e^{y_{1}^{*}(t)}}{\alpha(t)+\beta(t) e^{x_{1}^{*}(t)}+\gamma(t) e^{y_{1}^{*}(t)}} \\
& \left.-e_{1}(t)\left(u(t)-u^{*}(t)\right)\right]
\end{aligned}
$$




$$
\begin{aligned}
& =\operatorname{sgn}\left(x_{1}(t)-x_{1}^{*}(t)\right) \\
& \times\left[-b(t) e^{\xi(t)}\left(x_{1}(t)-x_{1}^{*}(t)\right)\right. \\
& -\frac{c(t) e^{y_{1}(t)}}{\alpha(t)+\beta(t) e^{x_{1}(t)}+\gamma(t) e^{y_{1}(t)}} \\
& +\frac{c(t) e^{y_{1}^{*}(t)}}{\alpha(t)+\beta(t) e^{x_{1}(t)}+\gamma(t) e^{y_{1}(t)}} \\
& -\frac{c(t) e^{y_{1}^{*}(t)}}{\alpha(t)+\beta(t) e^{x_{1}(t)}+\gamma(t) e^{y_{1}(t)}} \\
& +\frac{c(t) e^{y_{1}^{*}(t)}}{\alpha(t)+\beta(t) e^{x_{1}^{*}(t)}+\gamma(t) e^{y_{1}^{*}(t)}} \\
& \left.-e_{1}(t)\left(u(t)-u^{*}(t)\right)\right] \\
& \leq \operatorname{sgn}\left(x_{1}(t)-x_{1}^{*}(t)\right) \\
& \times\left[-b^{l} m_{1}\left(x_{1}(t)-x_{1}^{*}(t)\right)\right] \\
& -\frac{c(t)}{\alpha(t)+\beta(t) e^{x_{1}(t)}+\gamma(t) e^{y_{1}(t)}} \\
& \cdot e^{\eta(t)}\left(y_{1}(t)-y_{1}^{*}(t)\right) \\
& +\left(c ( t ) e ^ { y _ { 1 } ^ { * } ( t ) } \left[\beta(t)\left(e^{x_{1}(t)}-e^{x_{1}^{*}(t)}\right)\right.\right. \\
& \left.\left.+\gamma(t)\left(e^{y_{1}(t)}-e^{y_{1}^{*}(t)}\right)\right]\right) \\
& \times\left(\left(\alpha(t)+\beta(t) e^{x_{1}^{*}(t)}+\gamma(t) e^{y_{1}^{*}(t)}\right)\right. \\
& \left.\times\left(\alpha(t)+\beta(t) e^{x_{1}^{*}(t)}+\gamma(t) e^{y_{1}^{*}(t)}\right)\right)^{-1} \\
& -e_{1}(t)\left(u(t)-u^{*}(t)\right) \\
& \leq\left(\frac{c^{u} \beta^{u} M_{1} M_{2}}{\left(\alpha^{l}+\beta^{l} m_{1}+\gamma^{l} m_{2}\right)^{2}}-b^{l} m_{1}\right) \\
& \times\left|x_{1}(t)-x_{1}^{*}(t)\right| \\
& +\left(\frac{c^{u} \gamma^{u} M_{2}^{2}}{\left(\alpha^{l}+\beta^{l} m_{1}+\gamma^{l} m_{2}\right)^{2}}\right. \\
& \left.+\frac{c^{l} m_{2}}{\alpha^{u}+\beta^{u} M_{1}+\gamma^{u} M_{2}}\right) \\
& \times\left|y_{1}(t)-y_{1}^{*}(t)\right| \\
& +e_{1}^{u}\left|u(t)-u^{*}(t)\right| \text {. }
\end{aligned}
$$

Further, it follows that

$$
\begin{aligned}
D^{+} V_{2}(t)= & \operatorname{sgn}\left(u(t)-u^{*}(t)\right) \\
& \times\left(-d_{1}(t)\left(u(t)-u^{*}(t)\right)\right.
\end{aligned}
$$

$$
\begin{gathered}
+p_{1}(t)\left(e^{x_{1}(t-\tau)}-e^{x_{1}^{*}(t-\tau)}\right) \\
+p_{1}^{u}\left(e^{x_{1}(t)}-e^{x_{1}^{*}(t)}\right) \\
\left.-p_{1}(t)\left(e^{x_{1}(t-\tau)}-e^{x_{1}^{*}(t-\tau)}\right)\right) \\
\leq-d_{1}^{l}\left|u(t)-u^{*}(t)\right|+p_{1}^{u} M_{1}\left|x_{1}(t)-x_{1}^{*}(t)\right|, \\
D^{+} V_{3}(t)=\operatorname{sgn}\left(y_{1}(t)-y_{1}^{*}(t)\right) \\
\times\left[-\frac{r(t) e^{y_{1}(t)}}{e^{x_{1}(t)}+k(t)}+\frac{r(t) e^{y_{1}^{*}(t)}}{e^{x_{1}^{*}(t)}+k(t)}\right. \\
\left.\quad-e_{2}(t)\left(v(t)-v^{*}(t)\right)\right] \\
\leq-\frac{r^{l} m_{2}}{M_{1}+k^{u}}\left|y_{1}(t)-y_{1}^{*}(t)\right| \\
+\frac{r^{u} M_{1} M_{1}}{\left(m_{1}+k^{l}\right)^{2}}\left|x_{1}(t)-x_{1}^{*}(t)\right| \\
+e_{2}^{u}\left|\nu(t)-v^{*}(t)\right|, \\
D^{+} V_{4}(t) \leq-d_{2}^{l}\left|v(t)-v^{*}(t)\right|+p_{2}^{u} M_{2}\left|y_{1}(t)-y_{1}^{*}(t)\right| .
\end{gathered}
$$

Therefore, we have the following:

$D^{+} V(t)$

$$
\begin{aligned}
= & D^{+} V_{1}(T)+D^{+} V_{2}(T)+D^{+} V_{3}(T)+D^{+} V_{4}(T) \\
\leq & -\left(b^{l} m_{1}-p_{1}^{u} M_{1}-\frac{c^{u} \beta^{u} M_{1} M_{2}}{\left(\alpha^{l}+\beta^{l} m_{1}+\gamma^{l} m_{2}\right)^{2}}-\frac{r^{u} M_{1} M_{1}}{\left(m_{1}+k^{l}\right)^{2}}\right) \\
& \times\left|x_{1}(t)-x_{1}^{*}(t)\right| \\
& -\left(\frac{r^{l} m_{2}}{M_{1}+k^{u}}-\frac{c^{l} m_{2}}{\alpha^{u}+\beta^{u} M_{1}+\gamma^{u} M_{2}}\right. \\
& \left.\quad-\frac{c^{u} \gamma^{u} M_{2}^{2}}{\left(\alpha^{l}+\beta^{l} m_{1}+\gamma^{l} m_{2}\right)^{2}}-p_{2}^{u} M_{2}\right)\left|y_{1}(t)-y_{1}^{*}(t)\right| \\
& -\left(d_{1}^{l}-e_{1}^{u}\right)\left|u(t)-u^{*}(t)\right|-\left(d_{2}^{l}-e_{2}^{u}\right)\left|\nu(t)-v^{*}(t)\right| \\
\leq & -\Theta\left(\left|x_{1}(t)-x_{1}^{*}(t)\right|+\left|y_{1}(t)-y_{1}^{*}(t)\right|\right. \\
& \left.\quad+\left|u(t)-u^{*}(t)\right|+\left|\nu(t)-v^{*}(t)\right|\right) .
\end{aligned}
$$

Integrating the above inequality on internal $[0, t]$, it follows that, for $t \geq 0$,

$$
\begin{gathered}
V(t)+\Theta \int_{0}^{t}\left|x_{1}(t)-x_{1}^{*}(t)\right|+\left|y_{1}(t)-y_{1}^{*}(t)\right| \\
+\left|u(t)-u^{*}(t)\right|+\left|v(t)-v^{*}(t)\right| d s \\
\leq V(0)<+\infty .
\end{gathered}
$$


Then, for $t>0$, we obtain that

$$
\begin{aligned}
& \int_{0}^{t}\left|x_{1}(t)-x_{1}^{*}(t)\right|+\left|y_{1}(t)-y_{1}^{*}(t)\right| \\
& \quad+\left|u(t)-u^{*}(t)\right|+\left|\nu(t)-v^{*}(t)\right| d s \leq \frac{V(0)}{\Theta}<+\infty .
\end{aligned}
$$

By Lemma 7, we obtain

$$
\begin{array}{ll}
\lim _{t \rightarrow+\infty}\left|x_{1}(t)-x_{1}^{*}(t)\right|=0, & \lim _{t \rightarrow+\infty}\left|y_{1}(t)-y_{1}^{*}(t)\right|=0, \\
\lim _{t \rightarrow+\infty}\left|u(t)-u^{*}(t)\right|=0, & \lim _{t \rightarrow+\infty}\left|\nu(t)-v^{*}(t)\right|=0 .
\end{array}
$$

Then the solution of systems (4) and (5) is globally attractive.

Theorem 9. Suppose that all conditions of Theorem 8 hold; then there exists a unique almost periodic solution of systems (4) and (5).

Proof. According to Theorem 4, there exists a bounded positive solution $W(t)=\left(w_{1}(t), w_{2}(t), w_{3}(t), w_{4}(t)\right)^{T}$ of $(4)$ and (5). Then there exists a sequence $\left\{t_{k}^{\prime}\right\}, t_{k}^{\prime} \rightarrow \infty$ as $k \rightarrow \infty$, such that $\left(w_{1}\left(t+t_{k}^{\prime}\right), w_{2}\left(t+t_{k}^{\prime}\right), w_{3}\left(t+t_{k}^{\prime}\right), w_{4}\left(t+t_{k}^{\prime}\right)\right)^{T}$ is a solution of the following system:

$$
\begin{aligned}
& \dot{x}(t)=x(t)\left(a_{1}\left(t+t_{k}^{\prime}\right)-b\left(t+t_{k}^{\prime}\right) x(t)\right. \\
& -\frac{c\left(t+t_{k}^{\prime}\right) y(t)}{\alpha\left(t+t_{k}^{\prime}\right)+\beta\left(t+t_{k}^{\prime}\right) x(t)+\gamma\left(t+t_{k}^{\prime}\right) y(t)} \\
& \left.-e_{1}\left(t+t_{k}^{\prime}\right) u(t)\right) \text {, } \\
& \dot{u}(t)=-d_{1}\left(t+t_{k}^{\prime}\right) u(t)+p_{1}\left(t+t_{k}^{\prime}\right) x(t-\tau), \\
& \dot{y}(t)=y(t)\left(a_{2}\left(t+t_{k}^{\prime}\right)-\frac{r\left(t+t_{k}^{\prime}\right) y(t)}{x(t)+k\left(t+t_{k}^{\prime}\right)}\right. \\
& \left.-e_{2}\left(t+t_{k}^{\prime}\right) v(t)\right) \\
& \dot{v}(t)=-d_{2}\left(t+t_{k}^{\prime}\right) v(t)+p_{2}\left(t+t_{k}^{\prime}\right) y(t-\tau) .
\end{aligned}
$$

According to Theorem 3, we get that not only $\left\{\left(w_{1}(t+\right.\right.$ $\left.\left.\left.t_{k}^{\prime}\right), w_{2}\left(t+t_{k}^{\prime}\right), w_{3}\left(t+t_{k}^{\prime}\right), w_{4}\left(t+t_{k}^{\prime}\right)\right)^{T}\right\}$ but also $\left\{\left(\dot{w}_{1}\left(t+t_{k}^{\prime}\right), \dot{w}_{2}(t+\right.\right.$ $\left.\left.\left.t_{k}^{\prime}\right), \dot{w}_{3}\left(t+t_{k}^{\prime}\right), \dot{w}_{4}\left(t+t_{k}^{\prime}\right)\right)^{T}\right\}$ are uniformly bounded and equicontinuous. By Ascoli's theorem there exists a uniformly convergent subsequence $w_{i}\left(t+t_{k}\right) \subseteq w_{i}\left(t+t_{k}^{\prime}\right)(i=1,2,3,4)$ such that, for any $\varepsilon>0$, there exists a $K(\varepsilon)>0$ with the property that if $m, k \geq K(\varepsilon)$, then

$$
\left|w_{i}\left(t+t_{m}\right)-w_{i}\left(t+t_{k}\right)\right|<\varepsilon, \quad(i=1,2,3,4) .
$$

This is to say, $w_{i}\left(t+t_{k}\right)(i=1,2,3,4)$ are asymptotically almost periodic functions; hence there exist four almost periodic functions $P_{i}\left(t+t_{k}\right)(i=1,2,3,4)$ and four continuous functions $F_{i}\left(t+t_{k}\right)(i=1,2,3,4)$ such that

$$
w_{i}\left(t+t_{k}\right)=P_{i}\left(t+t_{k}\right)+F_{i}\left(t+t_{k}\right), \quad t \in R, i=1,2,3,4,
$$

where

$$
\begin{array}{r}
\lim _{k \rightarrow+\infty} P_{i}\left(t+t_{k}\right)=P_{i}(t), \quad \lim _{k \rightarrow+\infty} F_{i}\left(t+t_{k}\right)=0, \\
i=1,2,3,4
\end{array}
$$

$P_{i}(t)(i=1,2,3,4)$ are an almost periodic function.

Therefore,

$$
\lim _{k \rightarrow+\infty} w_{i}\left(t+t_{k}\right)=P_{i}(t), \quad(i=1,2,3,4) .
$$

On the other hand,

$$
\begin{aligned}
\lim _{k \rightarrow+\infty} \dot{w}_{i}\left(t+t_{k}\right) & =\lim _{k \rightarrow+\infty} \lim _{h \rightarrow 0} \frac{w_{i}\left(t+t_{k}+h\right)-w_{i}\left(t+t_{k}\right)}{h} \\
& =\lim _{h \rightarrow+\infty} \lim _{k \rightarrow 0} \frac{w_{i}\left(t+t_{k}+h\right)-w_{i}\left(t+t_{k}\right)}{h} \\
& =\lim _{h \rightarrow 0} \frac{P_{i}(t+h)-P_{i}(t)}{h}, \quad(i=1,2,3,4) .
\end{aligned}
$$

So $\dot{P}_{i}(t)(i=1,2,3,4)$ exist. Now we will prove that $\left(P_{1}(t), P_{2}(t), P_{3}(t), P_{4}(t)\right)^{T}$ is an almost periodic solution of system (4).

From properties of almost periodic function, there exits a sequence $\left\{t_{n}\right\},\left\{t_{n}\right\} \rightarrow \infty$ as $n \rightarrow \infty$, such that

$$
\begin{aligned}
& a_{i}\left(t+t_{n}\right) \longrightarrow a_{i}(t), d_{i}\left(t+t_{n}\right) \longrightarrow d_{i}(t), \\
& e_{i}\left(t+t_{n}\right) \longrightarrow e_{i}(t), p_{i}\left(t+t_{n}\right) \longrightarrow p_{i}(t), \\
&(i=1,2), \\
& b\left(t+t_{n}\right) \longrightarrow b(t), c\left(t+t_{n}\right) \longrightarrow c(t), \\
& r\left(t+t_{n}\right) \longrightarrow r(t), k\left(t+t_{n}\right) \longrightarrow k(t), \\
& \alpha\left(t+t_{n}\right) \longrightarrow \alpha(t), \beta\left(t+t_{n}\right) \longrightarrow \beta(t), \\
& \gamma\left(t+t_{n}\right) \longrightarrow \gamma(t),
\end{aligned}
$$

as $n \rightarrow \infty$ uniformly on $R$. 
It is easy to know that $w_{i}\left(t+t_{n}\right) \rightarrow P_{i}(t)(i=1,2,3,4)$ as $n \rightarrow \infty$, and then we have the following:

$$
\begin{aligned}
& \dot{P}_{1}(t) \\
& =\lim _{n \rightarrow+\infty} \dot{w}_{1}\left(t+t_{n}\right) \\
& =\lim _{n \rightarrow+\infty}\left[w _ { 1 } ( t + t _ { n } ) \left(a_{1}\left(t+t_{n}\right)-b\left(t+t_{n}\right) w_{1}\left(t+t_{n}\right)\right.\right. \\
& -\left(c\left(t+t_{n}\right) w_{3}\left(t+t_{n}\right)\right) \\
& \times\left(\alpha\left(t+t_{n}\right)+\beta\left(t+t_{n}\right) w_{1}\left(t+t_{n}\right)\right. \\
& \left.+\gamma\left(t+t_{n}\right) w_{3}\left(t+t_{n}\right)\right)^{-1} \\
& \left.\left.-e_{1}\left(t+t_{n}\right) w_{2}\left(t+t_{n}\right)\right)\right] \\
& =P_{1}(t)\left(a_{1}(t)-b(t) P_{1}(t)\right. \\
& \left.-\frac{c(t) P_{3}(t)}{\alpha(t)+\beta(t) P_{1}(t)+\gamma(t) P_{3}(t)}-e_{1}(t) P_{2}(t)\right) .
\end{aligned}
$$

By using a similar argument as that in the above, we have the following:

$$
\begin{gathered}
\dot{P}_{2}(t)=-d_{1}(t) P_{2}(t)+p_{1}(t) P_{1}(t-\tau), \\
\dot{P}_{3}(t)=P_{3}(t)\left(a_{2}(t)-\frac{r(t) P_{3}(t)}{P_{1}(t)+k(t)}-e_{2}(t) P_{4}(t)\right), \\
\dot{P}_{4}(t)=-d_{2}(t) P_{4}(t)+p_{2}(t) P_{3}(t-\tau) .
\end{gathered}
$$

This proves that $P_{i}(t)(i=1,2,3,4)$ is a nonnegative almost periodic solution of systems (4) and (5); by Theorem 8 , it follows that there exists a globally asymptotically stable nonnegative almost periodic solution of system (4). The proof is complete.

\section{An Example}

Consider the following system:

$$
\begin{gathered}
\dot{x}(t)=x(t)\left(4-2 x(t)-\frac{10 y(t)}{2+20 x(t)+20 y(t)}-2 u(t)\right), \\
\dot{u}(t)=-3 u(t)+\frac{1}{5} x(t-\tau), \\
\dot{y}(t)=y(t)\left(\frac{1}{10}-\frac{20 y(t)}{x(t)+23}-\frac{2}{5} v(t)\right), \\
\dot{v}(t)=-2 v(t)+2 y(t-\tau) .
\end{gathered}
$$

By a simple calculation, we check that all conditions in Theorems 8 and 9 are fulfilled. Therefore, by Theorems 8 and 9, system (48) has a unique globally asymptotically stable nonnegative almost periodic solution (see Figure 1).

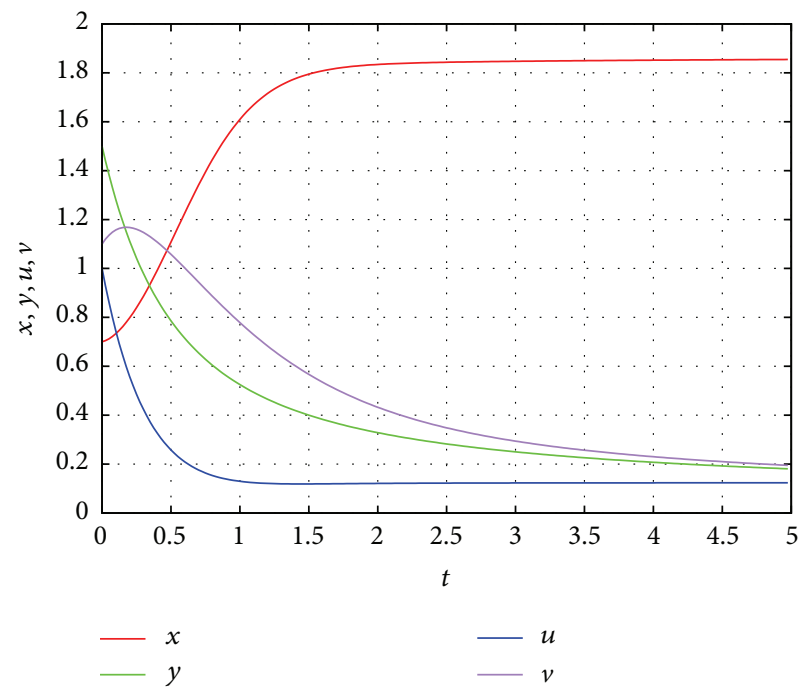

FIgURE 1: Dynamic behavior of system (48) with the initial $(x(0), y(0), u(0), v(0))^{T}=(0.7,1.5,1.0,1.1)^{T}$, for $\tau=0, t \in[0,5]$. From the figure, we could easily see that the solution $(x(t), y(t), u(t)$, $v(t))^{T}$ is asymptotic to the unique almost periodic solution of the system (48).

\section{Conflict of Interests}

The authors declare that there is no conflict of interests regarding the publication of this paper.

\section{Acknowledgment}

This work is supported by the NNSF of China (no. 11171085) and Hunan Provincial Natural Science Foundation of China (no. 10JJ6002).

\section{References}

[1] P. H. Leslie, "Some further notes on the use of matrices in population mathematics," Biometrika, vol. 35, no. 3-4, pp. 213249, 1948.

[2] S. B. Hsu and T. W. Huang, "Global stability for a class of predator-prey systems," SIAM Journal on Applied Mathematics, vol. 55, no. 3, pp. 763-783, 1995.

[3] P. H. Leslie and J. C. Gower, "The properties of a stochastic model for the predator-prey type of interaction between two species," Biometrika, vol. 47, pp. 219-234, 1960.

[4] A. Korobeinikov, "A Lyapunov function for Leslie-Gower predator-prey models," Applied Mathematics Letters, vol. 14, no. 6, pp. 697-699, 2001.

[5] M. A. Aziz-Alaoui and M. Daher Okiye, "Boundedness and global stability for a predator-prey model with modified LeslieGower and Holling-type II schemes," Applied Mathematics Letters, vol. 16, no. 7, pp. 1069-1075, 2003.

[6] H. F. Huo and W. T. Li, "Periodic solutions of delayed LeslieGower predator-prey models," Applied Mathematics and Computation, vol. 155, no. 3, pp. 591-605, 2004. 
[7] A. F. Nindjin, M. A. Aziz-Alaoui, and M. Cadivel, "Analysis of a predator-prey model with modified Leslie-Gower and Hollingtype II schemes with time delay," Nonlinear Analysis: Real World Applications, vol. 7, no. 5, pp. 1104-1118, 2006.

[8] L. Chen and F. Chen, "Global stability of a Leslie-Gower predator-prey model with feedback controls," Applied Mathematics Letters, vol. 22, no. 9, pp. 1330-1334, 2009.

[9] T. W. Zhang and X. R. Gan, "Existence and permanence of almost periodic solutions for Leslie-Gower predatorCprey model with variable delays," Electronic Journal of Differential Equations, vol. 2013, pp. 1-21, 2013.

[10] Z. M. Zhang, "Almost periodic solution of a modified LeslieGower predator-prey model with Beddington-DeAngelis functional response," Journal of Applied Mathematics, vol. 2013, Article ID 834047, 9 pages, 2013.

[11] F. Chen, Z. Li, and Y. Huang, "Note on the permanence of a competitive system with infinite delay and feedback controls," Nonlinear Analysis: Real World Applications, vol. 8, no. 2, pp. 680-687, 2007.

[12] X. Y. Dou and Y. K. Li, "Almost periodic solution for a foodlimited population model with delay and feedback control," International Journal of Computational and Mathematical Sciences, vol. 5, no. 4, pp. 174-179, 2011.

[13] M. Fan, K. Wang, and D. Jiang, "Existence and global attractivity of positive periodic solutions of periodic n-species LotkaVolterra competition systems with several deviating arguments," Mathematical Biosciences, vol. 160, no. 1, pp. 47-61, 1999. 


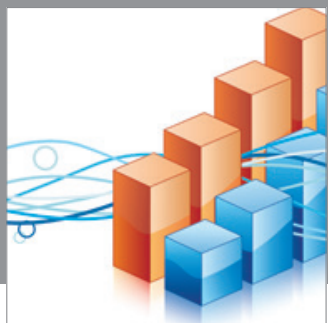

Advances in

Operations Research

mansans

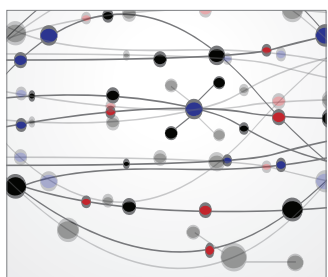

The Scientific World Journal
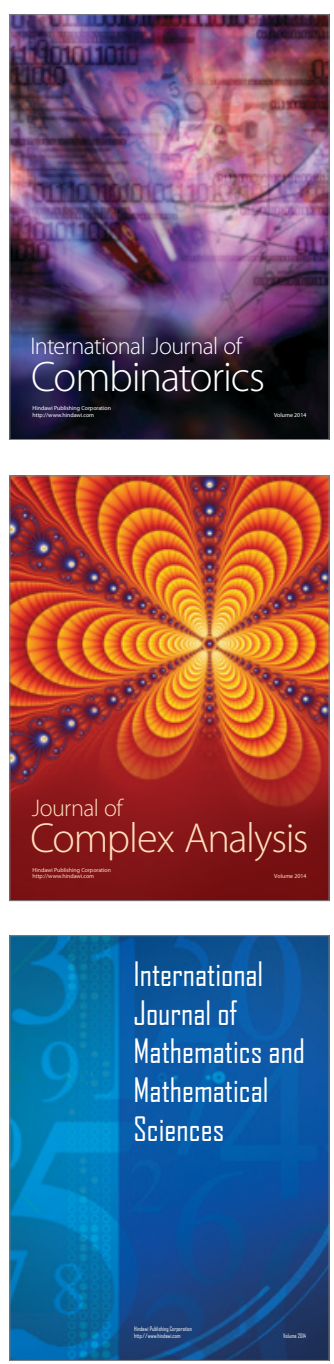
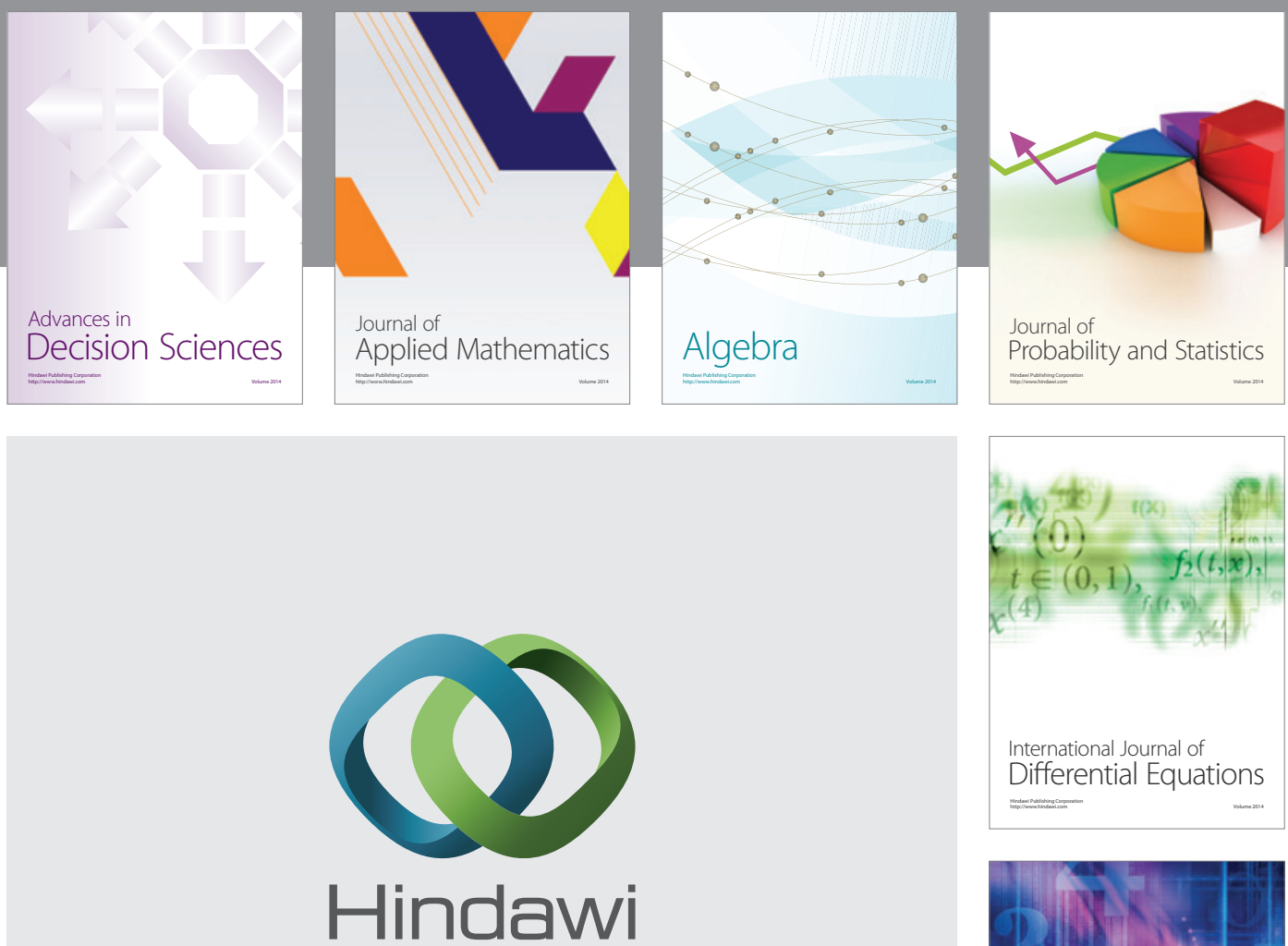

Submit your manuscripts at http://www.hindawi.com
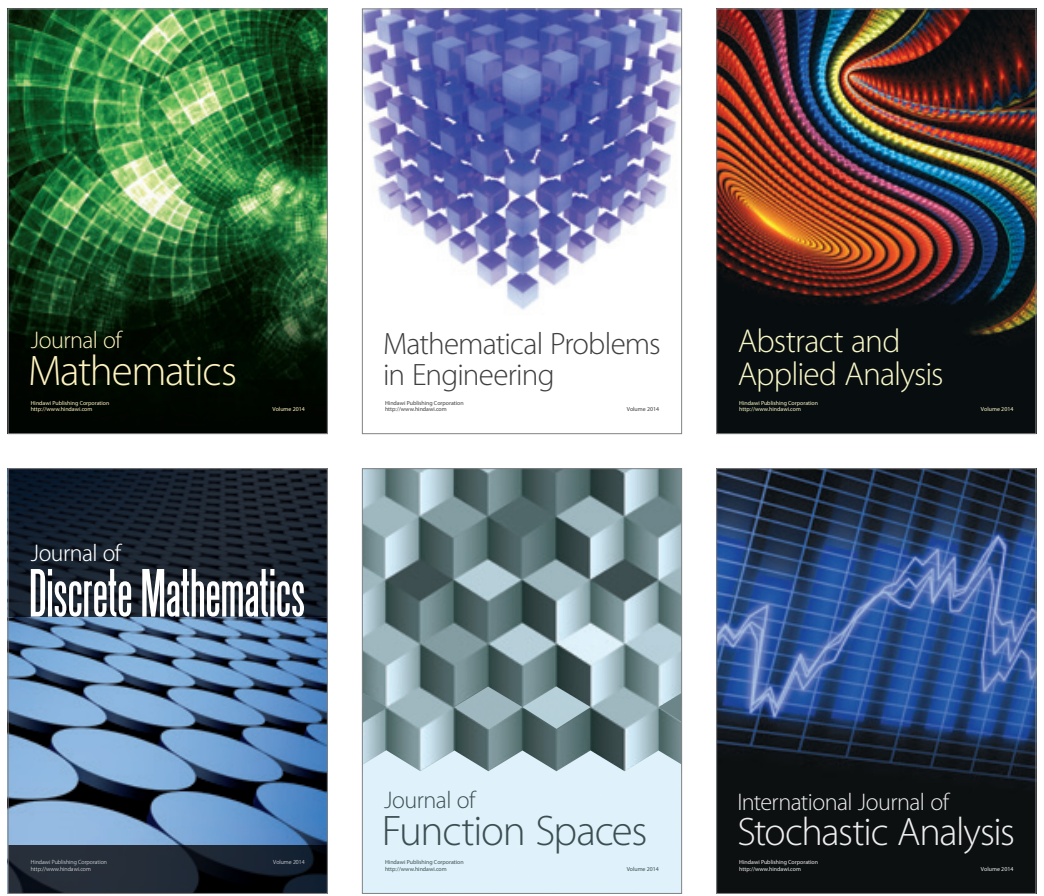

Journal of

Function Spaces

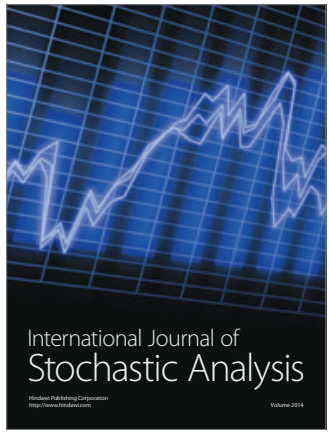

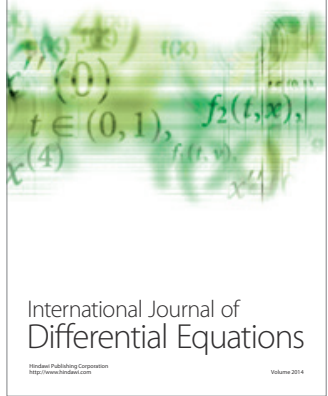
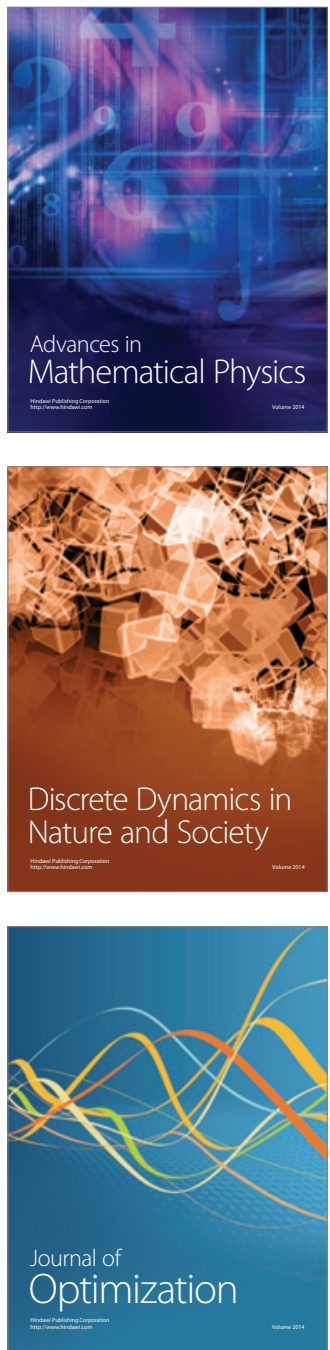